\title{
A Dynamic Market Clearing Price Mechanism with Multiple Demands*
}

Marilda Sotomayor $^{* *}$

\begin{abstract}
We propose a dynamic selling procedure for the generalization of the buyer-seller market game of Shapley and Shubik (1972) to the case where buyers can purchase more than one indivisible object, up to their quota, and have separable and additive utilities. This mechanism generalizes the auction studied by Demange et al. (1986), which in its turn is a generalization of the English auction to the multi-item case. It represents an enormous simplification of the restriction of the auction proposed by Gul and Stachetti (2000) to the linear case, by providing a different and simpler technique. Increasing the prices of all items of an overdemanded set chosen by the auctioneer always leads to the same price vector, namely the minimum competitive equilibrium price, in a finite number of steps. Consequently, the mechanism sells any two similar objects at the same price. When buyers are allowed to acquire the total amount of objects, the mechanism is strategyproof.
\end{abstract}

Keywords: Competitive Equilibrium Price, Optimal Matching, Overdemanded Set.

JEL Codes: C78, D78.

\footnotetext{
${ }^{*}$ Submitted in October 2003. Revised in February 2005. This work was partially supported by $\mathrm{CNPq}$ (National Council for Scientific and Technological Development), Brazil.

** USP. Email: marildas@usp.br

Brazilian Review of Econometrics

v. $25, \mathrm{n}^{\circ} 2$, pp. $267-286$ November 2005
} 


\section{Introduction}

In the model considered here, a set of indivisible objects is to be allocated to a set of buyers. Each buyer has a quota representing the maximum number of objects she can buy and places a monetary value on each object. Thus, if, say, buyer $i$, with quota three, values objects 1,2 and 3 in $a_{i 1}, a_{i 2}$ and $a_{i 3}$, respectively, and pays for them the prices $p_{1}, p_{2}$ and $p_{3}$, respectively, her payoff will be $\left(a_{i 1}-p_{1}\right)+\left(a_{i 2}-p_{2}\right)+\left(a_{i 3}-p_{3}\right){ }^{1}$ Therefore, this model is the simplest generalization of the buyer-seller market game treated in Shapley and Shubik (1972), in which the only restriction is that each buyer can purchase one object at most.

The natural economic question is then to determine (A) which objects will be allocated to each buyer and (B) at which prices they will be sold. The answer to these questions lies in the presumption that only Pareto efficient and envy-free feasible allocations will occur. The solution concept for this sort of market game, which captures this intuitive idea of fairness, is that of competitive equilibrium. ${ }^{2}$

Competitive equilibria always exist (see Sotomayor $(1992,1999)$ ). The question is then how to obtain these outcomes for the market treated here. The aim of the present paper is to find a dynamic mechanism that yields a competitive equilibrium price.

For the case where the quota of each buyer is one, a dynamic mechanism, which yields the minimum competitive equilibrium price, is presented by Demange et al. (1986). It is a version of the Hungarian algorithm (see, e.g., Dantzig (1963)) and a generalization of the English auction, which is strategically equivalent to the Vickrey's second-price auction.

The mechanism presented here extends the single-item English auction and the mechanism of Demange et al. Its main feature is that, similarly to these two auction procedures, it also yields the minimum competitive equilibrium price. However, unless the quotas of buyers are the total amount of objects, our mechanism is not strategy-proof. As a consequence of the fact that the final price is the minimum competitive equilibrium price, similar objects are sold for the same price. ${ }^{3}$ This property does not hold under an arbitrary clearing price mechanism.

\footnotetext{
${ }^{1}$ We may interpret the payoff of a buyer as follows, for example. Each buyer $i$ (whom we may think of as a broker, rather than as a final consumer of the object) has in hand an offer of $a_{i j}$ for each object $q_{j}$, from a client who wants any three of the objects, which might be cars, for instance, and will purchase them from the buyer at these prices.

${ }^{2}$ In the literature, a feasible allocation has been called fair if it is Pareto efficient and envy-free (see Alkan et al. (1991)).

${ }^{3}$ In 1981, Stherby Parke Bernet ran a sequential auction to sell seven identical licenses to use RCA's communications satellite for cable television broadcasts. The winning bids varied widely. The highest was $\$ 14.4$ million and the lowest was $\$ 10.7$ million. The FCC nullified the auction saying the procedure was "unjustly discriminatory" in charging different prices for the same service and ordered RCA to charge the same price to all. This story is told in PR Newswere, November 9, 1981; Christian Science Monitor, June 29, 1982 and Time, December 13, 1982, pp. 148.
} 
It can be easily seen, through a simple example of two sellers owing similar objects and one buyer with quota of two, that a competitive equilibrium does not need to sell similar objects for the same price. In fact, if, for instance, the buyer values both objects at $\$ 3$ and the reservation price of the objects is 1 , any price vector $\left(p_{1}, p_{2}\right)$ with $1 \leq p_{i} \leq 3$, for $i=1,2$, will be a competitive price, allocating both items to the buyer.

In terms of practical importance, our mechanism is simple enough so that it can be implemented in real auctions. In fact, at any step of the auction, given the prices announced by the auctioneer, buyers indicate their most preferred sets of objects at those prices (their demand sets) and quotas. Then, the auctioneer replicates each buyer the number of times of her quota and finds all demand structures (this will be further defined in the text) corresponding to the buyers' demands. This involves each copy of a buyer being associated with an object or with a set of objects in a precise way. No two copies have the same demand set. If there is some demand structure, such that it is possible to assign each copy of a buyer to an object in her demand set (which is equivalent to assigning every buyer to her most preferred set of objects at the given prices), then the auction ends. If no such assignment exists, a celebrated theorem due to Hall $(1935)^{4}$ implies that, for every demand structure, there exists a minimal overdemanded set, that is, an overdemanded set with the property that none of its proper subsets is overdemanded. A set of objects is overdemanded if the number of buyers demanding only items in this set is greater than the number of items in the set. Among all demand structures, the auctioneer chooses one that has the minimum number of minimal overdemanded sets. Next, he chooses a minimal overdemanded set in the demand structure selected and raises the price of each object in the set by one unit. All the other prices remain the same as in the previous stage. As in the one-to-one case, this algorithm ends in a finite number of steps.

A similar mechanism that applies to the case where utilities satisfy the monotonicity and gross substitute condition of Kelso and Crawford (1982) is presented by Gul and Stachetti (2000). The crucial difference between the two mechanisms, when applied to the linear case with quotas treated here, lies in how the auctioneer looks for overdemanded sets. The computation of these sets, even in quite simple situations, may involve a large number of steps if one employs the mechanism of Gul et al. It is the case of the simple example of four buyers, 1, 2, 3 and 4 , and six objects, $q_{1}, q_{2}, \ldots, q_{6}$. The quotas of the buyers are given by 3,2 , 1 and 1 , respectively. The values of the buyers to the objects are given by the following vectors: $v_{1}=(7,5,4,4,2,1), v_{2}=(5,3,1,0,2,1), v_{3}=(2,0,0,0,0,1)$

\footnotetext{
${ }^{4}$ Let $B$ and $C$ be two finite disjoint sets. For each $b$ in $B$, let $D_{b}$ be a subset of $C$. A simple assignment is an assignment of $C$ to $B$, such that each $b$ is assigned exactly one element $j$ of $C$, such that $j$ is in $D_{b}$, and each $j$ in $C$ is assigned to at most one element of $B$. Then, Hall's Theorem is: A simple assignment exists, if and only if, for every subset $B^{\prime}$ of $B$, the number of objects in $D\left(B^{\prime}\right)$ is at least as great as the number of buyers in $B^{\prime}$.
} 
and $v_{4}=(3,1,1,1,1,2)$, where the $i$ th coordinate of $v_{j}$ is the value of object $q_{i}$ to buyer $j$. The initial price is $p(0)=(0,0, \ldots, 0)$. At this price vector, and supposing the utilities of the buyers are additive, buyer 1 is indifferent between $\left\{q_{1}, q_{2}, q_{3}\right\}$ and $\left\{q_{1}, q_{2}, q_{4}\right\}$; buyer 2 wants $\left\{q_{1}, q_{2}\right\}$ and buyers 3 and 4 want $\left\{q_{1}\right\}$. It is not possible to meet the demands of every buyer giving to each of them her favorite set of objects, so the auctioneer has to find an overdemanded set. The procedure used by Gul and Stachetti to determine such a set involves, initially, the computation of four functions, referred to by the authors as "requirement functions," one for each object. The specification of the domain of any of these functions requires the determination of 64 elements; for each of these 64 elements it is necessary to compute two more numbers. Thus, at the end of the first step of the auction, $(64 \times 4)+(64 \times 2)=384$ computations will have already been made.

To see how simple our mechanism is, compared to that of Gul and Stachetti, consider the situation illustrated above. Replicate each buyer the number of times of her quota and determine the demand set of each copy. Since buyer 1 has a quota of 3 , buyer 2 has a quota of 2 , and buyers 3 and 4 have a quota of 1 , this procedure involves the simple computation of seven demand sets, one for each copy, straightforwardly derived from the list of preferences of the buyers over the objects, at the given price. There are two overdemanded sets: $\left\{q_{1}\right\}$ and $\left\{q_{2}\right\}$, both minimal. The auctioneer selects one of them and raises the price of the object by one unit. This defines the price to be announced by the auctioneer in the second step of the auction.

As for the techniques required in the proofs, while Gul and Stachetti use matroid theory and submodular optimization, one makes use of simple combinatorial arguments. This paper is organized as follows. In section 2, we describe the competitive model. The auction mechanism is presented in section 3. An illustrative example is given in subsection 3.1. Section 4 is devoted to the main results. Section 5 concludes the paper. Some of the proofs are left to the Appendix.

\section{The Competitive Market}

There is a set $B$ with $m$ bidders and a set $Q$ with $n$ indivisible objects. Each object $q$ has a reservation price of $c_{q}$. For simplicity, we will normalize and assume that $c_{q}=0$ for all objects $q$. Each bidder has a quota $r(b)$, representing the maximum number of objects she can buy. Without loss of generality we can consider $r(b) \leq n$. The value of object $q$ to bidder $b$ is $v_{b q} \geq 0$. (If $b$ buys $q$ at price $p_{q}$ she gets $v_{b q}-p_{q}$ ). We will assume that $Q$ contains an artificial "null object," 0 , whose value is zero to all bidders and whose price is always zero. The competitive market is then given by $(B, Q, v, r)$, where $v$ is the $m \times n$ matrix of values $v_{b q^{\prime} s}$, and $r$ is the vector of quotas.

When every buyer is allowed to have one object at most, the model is the Assignment Game of Shapley and Shubik (1972).

A feasible price vector $\mathbf{p}$, which will be referred to as price vector, is a 
function from $Q$ to $R^{+}$, such that $p(q) \equiv p_{q} \geq 0$. We will be assuming that the utility payoff of the buyers from acquiring one object at a given price is separable across pairs, in the sense that the $v_{b q^{\prime} s}$ do not depend on which other objects are acquired by buyer $b$. Thus, bidder $b$ prefers object $q$ to object $k$, at prices $p$, if $v_{b q}-p_{q}>v_{b k}-p_{k}$. She is indifferent between these two objects, if $v_{b q}-p_{q}=$ $v_{b k}-p_{k}$.

Given the price vector $p$, buyers also have preferences over sets of objects that can be allocated to them. An allowable set of objects for buyer $b$ contains $r(b)$ objects, some of which may be repetitions of the null object. A matching is an allocation of the objects to the buyers. If $b$ and $q$ are matched under $\mu$, we write $\mu(q)=b$ or $q \in \mu(b)$. If $q$ is not allocated to any buyer, we say that $q$ is unmatched at $\mu$. The null object may be matched to more than one buyer and more than once to the same buyer. If we consider that a bidder may be assigned to the object 0 as many times as she needs to fill up her quota, we can define a matching $\mu$ to be feasible, if each bidder is matched to an allowable set of objects for her and no non-null object is assigned to more than one bidder. The matching $\mu$ is optimal, if $\sum_{q \in Q} v_{\mu(q) q} \geq \sum_{q \in Q} v_{\mu^{\prime}(q) q}$ for all feasible $\mu^{\prime}$. Thus, an optimal matching is an efficient allocation of the objects. When all items are similar, an optimal matching allocates the objects to the bidders who value them most.

Since the quota restriction imposes a limit on the number of objects that a buyer $b$ may acquire, $b$ 's preferences under a feasible price vector are defined only over allowable sets of objects. The value of an allowable set of objects $S$ to buyer $b$ is the sum of the values of the objects in $S$ to $b$. Then, given a feasible price vector, the preferences of buyers are completely described by the numbers $v_{b q}$ : For any two allowable sets of objects $S$ and $S^{\prime}$, buyer $b$ prefers $S$ to $S^{\prime}$ at prices $p$, if her total payoff when she buys $S$ is greater than her total payoff when she buys $S^{\prime}$. She is indifferent between these two sets if she gets the same total payoff with both sets. Object $q$ is acceptable to buyer $b$ at prices $p$, if $b$ likes $q$ at least as well as the null-object under prices $p$.

Under the structure of preferences we are assuming, each buyer $b$ can determine which allowable sets of objects she would most prefer to buy at a given price vector $p$ by looking at only her list of preferences $P_{b}(p)$. We denote the set of all such sets by $\mathbf{D}_{\mathbf{b}}(\mathbf{p})$ and call it the demand set of $\mathbf{b}$ at prices $\mathbf{p}$. (Note that $D_{b}(p)$ is never empty, because there is always the option of buying $r(b)$ copies of the null object. Note also that, if $S \in D_{b}(p)$, then every element of $S$ is acceptable to $\left.b\right)$. Hence, $S$ and $S^{\prime}$ are in $D_{b}(p)$, if $\sum_{q \in S}\left(v_{b q}-p_{q}\right)=\sum_{q \in S^{\prime}}\left(v_{b q}-p_{q}\right)>\sum_{q \in S^{\prime \prime}}\left(v_{b q}-p_{q}\right)$ for all allowable sets $S^{\prime \prime} \notin D_{b}(p)$.

Definition 1. The feasible price vector $p$ is called competitive for $M$, if there is a feasible matching $\mu$ such that, if $\mu(b)=S$, then $S$ is in $D_{b}(p)$. 
Therefore, at competitive prices $p$, each buyer is assigned to a set of objects in her demand set. The matching $\mu$ is said to be compatible with $p$. A matching $\mu$ is called competitive if it is compatible with a competitive price.

Definition 2. The pair $(p, \mu)$ is a competitive equilibrium for $M$, if $p$ is competitive, $\mu$ is compatible with $p$, and $p_{q}=0$ if object $q$ is left unsold. If $(p, \mu)$ is a competitive equilibrium, $p$ will be called an equilibrium price vector.

Thus, at a competitive equilibrium, not only does every buyer get a set of objects in her demand set, but also no unsold object has a price higher than its reservation price. If $(p, \mu)$ is a competitive equilibrium, $p$ will be called an equilibrium price vector. In Sotomayor (1999), it was proved that a matching is optimal if and only if it is competitive. This result was proved by Shapley and Shubik (1972) for the case of unitary demands.

Definition 3. The competitive equilibrium $(p, \mu)$ is a minimum (respectively, maximum) competitive equilibrium for $M$, if $p_{q} \leq p_{q}^{\prime}$ (respectively, if $p_{q} \geq p_{q}^{\prime}$ ) for all objects $q$ and all equilibrium price vectors $p^{\prime}$.

\section{The Auction Mechanism}

In this section we will describe a dynamic mechanism for the competitive market $M$, and will see that it produces, in a finite number of steps, a competitive price. It will be proved in the next section that the resulting allocation is the minimum competitive equilibrium for $M$. Before doing that, we need some preliminaries.

Given the price vector $p$, the preferences of each buyer b over individual objects can be represented by an ordered list of preferences, $P_{b}(p)$, on the set of objects. That is, bidder $b^{\prime}$ s preferences might be of the form:

$$
P_{b}(p)=q_{1}, q_{2}, q_{3}, 0, q_{4}
$$

indicating that she prefers $q_{1}$ to $q_{2}, q_{2}$ to $q_{3}$, any of these objects to 0 , but prefers not to fill her quota to buy $q_{4}$. Given the prices $p$, a bidder $b$ may be indifferent among several possible objects. Brackets in the preference list will denote this, so, for example, the list

$$
P_{b^{\prime}}(p)=\left[q_{1}, q_{2}\right], q_{3}, q_{4}\left[q_{5}, q_{6}, q_{7}\right], 0
$$

indicates that bidder $b^{\prime}$ is indifferent between $q_{1}$ and $q_{2}$, prefers any of these objects to $q_{3}$, and so on.

Given the price vector $p$, the bid of $\mathbf{b}$ at $\mathbf{p}, \mathbf{B}_{\mathbf{b}}(\mathbf{p})$, is a truncation of the preference list $P_{b}(p)$, so that it contains exactly all $S \in D_{b}(p)$. To fix ideas, consider that we have ten non-null objects, $r(b)=8$ and $v_{b}=(7,7,5,4,2,2,2,1,1,1,0)$. Suppose $p=(0,0, \ldots, 0)$. Then, $b$ 's preferences over individual objects, at price $p$, are given by: 


$$
P_{b}(p)=\left[q_{1}, q_{2}\right], q_{3}, q_{4},\left[q_{5}, q_{6}, q_{7}\right],\left[q_{8}, q_{9}, q_{10}\right], q_{11}, q_{12}, 0 .
$$

The demand set of $b$ at $p$ is:

$$
\begin{aligned}
D b(p)= & \left\{\left\{q_{1}, q_{2}, q_{3}, q_{4}, q_{5}, q_{6}, q_{7}, q_{8}\right\},\right. \\
& \left\{q_{1}, q_{2}, q_{3}, q_{4}, q_{5}, q_{6}, q_{7}, q_{9}\right\},\left\{q_{1}, q_{2}, q_{3}, q_{4}, q_{5}, q_{6}, q_{7}, q_{10}\right\}
\end{aligned}
$$

Then, $B_{b}(p)=\left[q_{1}, q_{2}\right], q_{3}, q_{4},\left[q_{5}, q_{6}, q_{7}\right],\left[q_{8}, q_{9}, q_{10}\right]$.

Remark 1. From the definition of $B_{b}(p)$, it follows that $q \in B_{b}(p)$, if and only if the number of objects preferred to $q$ by $b$ at prices $p$ is less than $r(b)$.

The strict bids of $\mathbf{b}$ at prices $\mathbf{p}$ are all lists of strict preferences that can be derived from $B_{b}(p)$ by using some tie-breaking rule. In the example above the strict bids are:

$$
\begin{aligned}
B_{b}^{s}(p) & =q_{1}, q_{2}, q_{3}, q_{4}, q_{5}, q_{6}, q_{7}, q_{8}, q_{9}, q_{10} \\
B_{b}^{s^{\prime}}(p) & =q_{1}, q_{2}, q_{3}, q_{4}, q_{5}, \mathbf{q}_{\mathbf{7}}, \mathbf{q}_{\mathbf{6}}, q_{8}, q_{9}, q_{10} \\
B_{b}^{s^{\prime \prime}}(p) & =\mathbf{q}_{\mathbf{2}}, \mathbf{q}_{\mathbf{1}}, q_{3}, q_{4}, \mathbf{q}_{\mathbf{6}}, \mathbf{q}_{\mathbf{5}}, q_{7}, q_{9}, q_{8}, q_{10}, \text { etc. }
\end{aligned}
$$

For each strict bid, $B_{b}^{s}(p)$, there is a corresponding demand structure $\mathbf{A}_{\mathbf{b}}(\mathbf{p})=$ $\left\{\mathbf{A}_{\mathbf{b}, \mathbf{1}}(\mathbf{p}), \ldots, \mathbf{A}_{\mathbf{b}, \mathbf{r}(\mathbf{b})}(\mathbf{p})\right\}$. We can think of each buyer $b$ being replicated $r(b)$ times: $(b, 1),(b, 2), \ldots,(b, r(b))$. The $i$ th copy $(b, i)$ demands the $i$ th object of $B_{b}^{s}(p)$, if $i<r(b)$, and $(b, r(b))$ demands the objects of $B_{b}^{s}(p)$ that were not demanded by the previous copies. This defines the set $\mathbf{A}_{\mathbf{b}, \mathbf{i}}(\mathbf{p})$. Formally,

$A_{b, i}(p)$ is the set formed by the $i$ th object in $B_{b}^{s}(p)$, if $i<r_{b}$;

$A_{b, i}(p)$ is the set formed by the objects in $B_{b}^{s}(p)$ which are not in $\left[A_{b, 1}(p) \cup\right.$ $\left.\ldots \cup A_{b, r(b)-1}(p)\right]$, if $i=r_{b}$.

The set of all $A_{b^{\prime} s}(p)$ will be called a demand structure at $\mathbf{p}$ and will be denoted by $A(p)$.

Thus, in the example above, the demand structure for $b$ at prices $p$ corresponding to $B_{b}^{s}(p)$ is: $A_{b, 1}(p)=\left\{q_{1}\right\}, A_{b, 2}(p)=\left\{q_{2}\right\}, A_{b, 3}(p)=\left\{q_{3}\right\}, A_{b, 4}(p)=\left\{q_{4}\right\}$, $A_{b, 5}(p)=\left\{q_{5}\right\}, A_{b, 6}(p)=\left\{q_{6}\right\}, A_{b, 7}(p)=\left\{q_{7}\right\}$ and $A_{b, 8}(p)=\left\{q_{8}, q_{9}, q_{10}\right.$.

For each demand structure $A(p)$, we say that copy $(b, i)$ is a loyal demander of $\mathbf{S}$ if $A_{b, i}(p) \subseteq S$.

Definition 4. Given the price vector $p$, we will say that the set $S \subseteq Q$ is overdemanded for the demand structure $A(p)$, if there is a set $T$ of loyal demanders of $S$, such that $|T|>|S|$.

The overdemanded set $S$ is said to be minimal, if no proper subset of $S$ is overdemanded. Thus, if $b$ has quota $r(b)=1$ and $A_{b, 1}(p)=\left\{q_{3}, q_{4}\right\} ; b^{\prime}$ has quota $r\left(b^{\prime}\right)=2, A_{b^{\prime}, 1}(p)=\left\{q_{3}\right\}$ and $A_{b^{\prime}, 2}(p)=\left\{q_{4}\right\}$; and $b^{\prime \prime}$ has quota $r\left(b^{\prime \prime}\right)=2$, $A_{b^{\prime \prime}, 1}(p)=\left\{q_{1}\right\}$ and $A_{b^{\prime \prime}, 2}(p)=\left\{q_{3}\right\}$, then, $T=\left\{(b, 1),\left(b^{\prime}, 1\right),\left(b^{\prime}, 2\right),\left(b^{\prime \prime}, 2\right)\right\}$ is a 
set of loyal demanders of $S=\left\{q_{3}, q_{4}\right\}$. The set $S$ is overdemanded, but it is not minimal. In fact, the set $S^{\prime}=\left\{q_{3}\right\}$ is overdemanded by $T^{\prime}=\left\{\left(b^{\prime}, 1\right),\left(b^{\prime \prime}, 2\right)\right\}$. In this example, $S^{\prime}$ is a minimal overdemanded set.

Remark 2. It follows from the definition of competitive equilibrium that, if $p$ is a competitive equilibrium price, then each buyer $b$ can be matched to some of her favorite set of objects at prices $p$. Therefore, there is some demand structure $A(p)$ for which each copy $(b, i)$ can be matched to exactly one object $q$, such that $q \in A_{b, i}(p)$, and no $q \neq 0$ is matched twice. Hence, there is no overdemanded set for $A(p)$.

If $p$ is not a competitive equilibrium price, then there is no way to match each buyer to any of her favorite set of objects under a feasible matching. This means that every demand structure $A(p)$ has an overdemanded set. This is a consequence of Hall's Theorem (See Hall (1935), Gale (1960)).

Now, we can describe our auction mechanism. We will take all prices and valuations as integers.

Step (1): The auctioneer announces an initial price vector, $p(1)$, equal to the vector $c$ of reservation prices, which we are supposing to be $(0, \ldots, 0)$. Each bidder $b$ "bids" by announcing $B_{b}(1) \equiv B_{b}(p(1))$.

Step $(\mathbf{t}+\mathbf{1})$ : After the bids are announced, the auctioneer determines all the demand structures at $p(t)$, using all possible strict bids. If there is some demand structure $A(t) \equiv A(p(t))$, for which it is possible to match each pair $(b, i)$ to an object $q \in A_{(b, i)}(t)$, so that no non-null object is matched twice, the algorithm stops. If no such demand structure exists, Hall's Theorem implies that there is some overdemanded set for every demand structure.

Then, the auctioneer chooses some demand structure that has the minimum number of minimal overdemanded sets among all demand structures. (This corresponds to the choice of a tie-breaking rule). Next, he selects a minimal overdemanded set for the demand structure chosen and raises the price of each object in the set by one unit. All other prices remain at level $p(t)$. This defines $p(t+1)$.

It is clear that the algorithm stops at some step, because, as soon as the price of an object becomes higher than any bidder's valuation for it, no bidder can demand it. It follows from the construction of the algorithm that the final price is a competitive price vector for market $(B, Q, v, r)$. What is less clear is that this algorithm yields the same price, independent of the demand structure selected by the auctioneer. We will prove this fact in section 4 by showing that the price obtained in the algorithm is the minimum equilibrium price vector.

\subsection{An example}

The following example illustrates our auction mechanism. There are four buyers, buyers $1,2,3$ and 4 , and six objects, $q_{1}, q_{2}, \ldots, q_{6}$. The maximum number of 
objects that each buyer can purchase is given by $3,2,1$ and 1 , respectively. These numbers define the quotas of the buyers. The values of the buyers to the objects are given by the following vectors: $v_{1}=(7,5,4,4,2,1,0), v_{2}=(5,3,1,0,2,1,0)$, $v_{3}=(2,0,0,0,0,1,0)$ and $v_{4}=(3,1,1,1,1,2,0)$, where the $i$ th coordinate of $v_{j}$ is the value of object $q_{i}$ to buyer $j$. The value of a set of objects to a buyer is the sum of the values of these objects to the given buyer, assuming that the number of objects in the set does not exceed the quota of the buyer.

Step 1. $p(1)=(0,0, \ldots, 0)$. The bids of the buyers at $p(1)$ are: $B_{1}(1)=$ $q_{1}, q_{2},\left[q_{3}, q_{4}\right] ; B_{2}(1)=q_{1}, q_{2} ; B_{3}(1)=B_{4}(1)=q_{1}$. There are two strict bids for buyer 1 and only one demand structure given by: $A_{1,1}(1)=\left\{q_{1}\right\}, A_{1,2}(1)=\left\{q_{2}\right\}$, $A_{1,3}(1)=\left\{q_{3}, q_{4}\right\} ; A_{2,1}(1)=\left\{q_{1}\right\}, A_{2,2}(1)=\left\{q_{2}\right\}, A_{3,1}(1)=\left\{q_{1}\right\}$ and $A_{4,1}(1)=$ $\left\{q_{1}\right\}$. We have seven copies demanding only four objects, so it is not possible to find a competitive matching. However $\left\{q_{1}, q_{2}, q_{3}, q_{4}\right\}$ is not minimal. There are two minimal overdemanded sets: $\left\{q_{1}\right\}$ and $\left\{q_{2}\right\}$. Suppose the auctioneer chooses $\left\{q_{1}\right\}$. Then, he raises the price of $\left\{q_{1}\right\}$ by one unit.

Step 2. $p(2)=(1,0, \ldots, 0)$. Then, $B_{1}(2)=q_{1}, q_{2},\left[q_{3}, q_{4}\right] ; B_{2}(2)=q_{1}, q_{2} ;$ $B_{3}(2)=B_{4}(2)=\left[q_{1}, q_{6}\right]$. As in the previous step, there is only one demand structure. The only difference is that now $A_{3,1}(2)=\left\{q_{1}, q_{6}\right\}$ and $A_{4,1}(2)=\left\{q_{1}, q_{6}\right\}$. There are two minimal overdemanded sets: $\left\{q_{1}\right\}$ and $\left\{q_{2}\right\}$. Suppose the auctioneer chooses $\left\{q_{1}\right\}$. As a result, he raises the price of $q_{1}$ by one unit.

Step 3. $p(3)=(2,0, \ldots, 0)$. Then, $B_{1}(3)=\left[q_{1}, q_{2}\right],\left[q_{3}, q_{4}\right] ; B_{2}(3)=\left[q_{1}, q_{2}\right]$; $B_{3}(3)=B_{4}(3)=q_{6}$. There are four demand structures, $A^{1}, A^{2}, A^{3}$ and $A^{4}$, given by:

$$
\begin{array}{llll}
A_{1,1}^{1}(3)=\left\{q_{1}\right\}, & A_{1,1}^{2}(3)=\left\{q_{2}\right\}, & A_{1,1}^{3}(3)=\left\{q_{1}\right\}, & A_{1,1}^{4}(3)=\left\{q_{2}\right\}, \\
A_{1,2}^{1}(3)=\left\{q_{2}\right\}, & A_{1,2}^{2}(3)=\left\{q_{1}\right\}, & A_{1,2}^{3}(3)=\left\{q_{2}\right\}, & A_{1,2}^{4}(3)=\left\{q_{1}\right\}, \\
A_{1,3}^{1}(3)=\left\{q_{3}, q_{4}\right\}, & A_{1,3}^{2}(3)=\left\{q_{3}, q_{4}\right\}, & A_{1,3}^{3}(3)=\left\{q_{3}, q_{4}\right\}, & A_{1,3}^{4}(3)=\left\{q_{3}, q_{4}\right\}, \\
A_{2,1}^{1}(3)=\left\{q_{1}\right\}, & A_{2,1}^{2}(3)=\left\{q_{1}\right\}, & A_{2,1}^{3}(3)=\left\{q_{2}\right\}, & A_{2,1}^{4}(3)=\left\{q_{2}\right\}, \\
A_{2,2}^{1}(3)=\left\{q_{2}\right\}, & A_{2,2}^{2}(3)=\left\{q_{2}\right\}, & A_{2,2}^{3}(3)=\left\{q_{1}\right\}, & A_{2,2}^{4}(3)=\left\{q_{1}\right\}, \\
A_{3,1}^{1}(3)=\left\{q_{6}\right\}, & A_{3,1}^{2}(3)=\left\{q_{6}\right\}, & A_{3,1}^{3}(3)=\left\{q_{6}\right\}, & A_{3,1}^{4}(3)=\left\{q_{6}\right\}, \\
A_{4,1}^{1}(3)=\left\{q_{6}\right\}, & A_{4,1}^{2}(3)=\left\{q_{6}\right\}, & A_{4,1}^{3}(3)=\left\{q_{6}\right\}, & A_{4,1}^{4}(3)=\left\{q_{6}\right\} .
\end{array}
$$

There are three minimal overdemanded sets in each demand structure: $\left\{q_{1}\right\}$, $\left\{q_{2}\right\}$ and $\left\{q_{6}\right\}$. Suppose the auctioneer chooses $\left\{q_{2}\right\}$. As a result, he raises the price of $q_{2}$ by one unit.

Step 4. $p(4)=(2,1, \ldots, 0)$. Then, $B_{1}(4)=q_{1},\left[q_{2}, q_{3}, q_{4}\right] ; B_{2}(4)=q_{1},\left[q_{2}, q_{5}\right]$; $B_{3}(4)=B_{4}(4)=q_{6}$. The reader is invited to see that there are three demand structures and two minimal overdemanded sets in each of them: $\left\{q_{1}\right\}$ and $\left\{q_{6}\right\}$. Suppose the auctioneer chooses $\left\{q_{1}\right\}$. As a result, he raises the price of $q_{1}$ by one unit.

Step 5. $p(5)=(3,1, \ldots, 0)$. Then, $B_{1}(5)=\left[q_{1}, q_{2}, q_{3}, q_{4}\right] ; B_{2}(5)=\left[q_{1}, q_{2}, q_{5}\right]$; $B_{3}(5)=B_{4}(5)=q_{6}$. There are eight possibilities of demand for buyer 1 , three 
possibilities for buyer 2 and one possibility for buyers 3 and 4, so there are twentyfour demand structures. There are three groups of minimal overdemanded sets found in these demand structures: $\left\{\left\{q_{1}\right\},\left\{q_{6}\right\}\right\},\left\{\left\{q_{2}\right\},\left\{q_{6}\right\}\right\}$, and $\left\{q_{6}\right\}$. The auctioneer must choose a demand structure with the minimum number of minimal overdemanded sets. Then the auctioneer chooses one with minimal overdemanded set $\left\{q_{6}\right\}$. As a result, he raises the price of $q_{6}$ by one unit.

Step 6. $p(6)=(3,1,0,0,0,1,0)$. Then, $B_{1}(6)=\left[q_{1}, q_{2}, q_{3}, q_{4}\right] ; B_{2}(6)=$ $\left[q_{1}, q_{2}, q_{5}\right] ; B_{3}(6)=\left[q_{3}, q_{4}, q_{5}, q_{6}, 0\right]$ and $B_{4}(6)=\left[q_{3}, q_{4}, q_{5}, q_{6}\right]$. There is a competitive matching that matches buyer 1 to $\left\{q_{2}, q_{3}, q_{4}\right\}$, buyer 2 to $\left\{q_{1}, q_{5}\right\}$, buyer 3 to the null-object and buyer 4 to $q_{6}$. Therefore, the final price is $(3,1,0,0,0,1,0)$.

\section{Main Results}

In this section, we demonstrate that the final price $p$ yielded by the auction mechanism is the minimum competitive price. Next, we state (and prove in the Appendix) that the final matching $\mu$ can be chosen so that $(p, \mu)$ is an equilibrium. In Proposition 1, we demonstrate that similar objects are sold for the same price. This property is strongly related to the minimality of the competitive price $p$.

In Sotomayor (1992) it is proved that the one-shot mechanism that yields the minimum competitive equilibrium is manipulable by the buyers. When all gross substitute preferences are allowed, Gul and Stachetti (2000) prove that no ascending price auction mechanism can be efficient and strategy proof. Under the quota restriction and the assumption that the utilities are additive, Proposition 2 proves that our dynamic mechanism is not strategy-proof. However, when the quota of each buyer is the total number of objects, Proposition 3 shows that truth telling is a dominant strategy for the buyers. Indeed, in this case, the payment of a bidder under the minimum competitive equilibrium price coincides with his payment under the Vickrey-Clarke-Groves mechanism, which is strategy-proof.

We will denote the minimum competitive price by y and the price vector obtained in the auction by $p$. For each step $t$ of the auction denote $U(t) \equiv\{q \in$ $\left.Q ; p_{q}(t)=y_{q}\right\}$. The fact that $p$ is competitive implies that $p \geq y$. We also have that $p(1)=c$, so $p(1) \leq y$. Therefore, since we are working with all integers, there is at least one-step $t$ of the auction such that $U(t) \neq \phi$ and $p(t) \leq y$.

From the competitiveness of $y$, it follows that there is some demand structure for $y$ with no overdemanded set. Choose one of such demand structures and call it $A^{*}(y)$.

We need the following results whose proofs are left to the Appendix.

Lemma 1. Let $t$ be some step of the auction at which the set $U(t) \neq \phi$ and $p(t) \leq$ $y$. Let $A(t)$ be any demand structure at step $t$. Let $T^{\prime}=\left\{(b, i) ; A_{b, i}(t) \cap U(t) \neq \phi\right\}$. Suppose that $T^{\prime} \neq \phi$. Then, there is some demand structure $A^{\prime}(t)$, such that for each $(b, i) \in T^{\prime}$, there exists some $(b, j)$, with $A_{b, j}^{*}(y) \subseteq U(t)$, and such that $A_{b, j}^{*}(y)=A_{b, i}^{\prime}(t)$ if $i \neq r(b)$ and $A_{b, j}^{*}(y) \subseteq A_{b, i}^{\prime}(t)$, otherwise. Furthermore, 
$A_{b, i}^{\prime}(t)=A_{b, i}(t)$ for all $(b, i) \notin T^{\prime}$.

Lemma 2. Let $t$ be some step of the auction at which the set $U(t) \neq \phi$ and $p(t) \leq y$. Let $A(t)$ be any demand structure at step $t$ under price $p(t)$. Let $A^{\prime}(t)$ be defined as in Lemma 1. Then, $A^{\prime}(t)$ has no minimal overdemanded set containing elements of $U(t)$.

Lemma 3. Let $t$ be some step of the auction at which the set $U(t) \neq \phi$ and $p(t) \leq y$. Let $A(t)$ be any demand structure at step $t$ under price $p(t)$. Let $A^{\prime}(t)$ be defined as in Lemma 1. Then, every minimal overdemanded set for $A^{\prime}(t)$, if any, is a minimal overdemanded set for $A(t)$.

Theorem 1. Price vector $p$ is the minimum competitive price for $M$.

Proof: Suppose by way of contradiction that $p$ is not the minimum competitive price. Then, $p>y$. Let $t$ be the last step of the auction at which $p(t) \leq y$ and let $S_{1}=\left\{q \in Q ; p_{q}(t+1)>y_{q}\right\}$. Then, $S_{1} \neq \phi$. Since we are working with all integers, $S_{1} \subseteq U(t)$. Let $A(t)$ be the demand structure selected by the auctioneer at prices $p(t)$ which has the minimum number of minimal overdemanded sets. Let $S$ be the minimal overdemanded set for $A(t)$, whose prices are raised at stage $t+1$. Thus, $S=\left\{q \in Q ; p_{q}(t+1)>p_{q}(t)\right\}$, so $S_{1}=S \cap U(t)$, and so $S \cap U(t) \neq \phi$.

By Lemma 1 and Lemma 2, there is some demand structure $A^{\prime}(t)$, defined from $A(t)$ and $A^{*}(y)$, which has no minimal overdemanded set containing some element of $U(t)$. Then, $S$ is not a minimal overdemanded set for $A^{\prime}(t)$. On the other hand, Lemma 3 asserts that every minimal overdemanded set for $A^{\prime}(t)$, if any, is a minimal overdemanded set for $A(t)$. Therefore, $A^{\prime}(t)$ has fewer minimal overdemanded sets than $A(t)$, contradicting the rules of the auction. Hence, $p=y$ and $p$ is the minimum competitive price.

Theorem 2. If $p$ is the minimum competitive price, then there is a matching $\mu$ such that $(p, \mu)$ is an equilibrium.

Proof. See the Appendix.

Proposition 1. Let $p$ be the minimum competitive equilibrium price. Let $j, k \in Q$, such that $v_{b j}=v_{b k}$ for all $b \in B$. Then, $p_{j}=p_{k}$.

Proof. Let $\mu$ be some competitive matching. Suppose, by way of contradiction, that $p_{j} \neq p_{k}$. Without loss of generality, it can be assumed that $p_{j}>p_{k} \geq 0$. Then, object $j$ must be allocated to some buyer $b^{*}$ and $v_{b^{*} j}-p_{j}<v_{b^{*} j}-p_{k}=$ $v_{b^{*} k}-p_{k}$. The competitiveness of $p$ implies that $\{j, k\} \subseteq \mu\left(b^{*}\right)$. We claim that the price vector $p^{*}$ is also competitive, where $p_{j}^{*}=p_{k}^{*}=p_{k}$ and $p_{q}^{*}=p_{q} \forall q \notin\{j, k\}$. We have to show that, if $\mu(q)=b$, then $v_{b q}-p_{q}^{*} \geq v_{b q^{\prime}}-p_{q^{\prime}}^{*}$, for every $q^{\prime} \notin \mu(b)$. (This is equivalent to requiring that $\mu(b)$ be in $D_{b}\left(p^{*}\right)$ ). We have two cases. 
Case 1. $q \in\{j, k\}$. Then, $b=b^{*}$ and for all $q^{\prime} \notin \mu\left(b^{*}\right)$, we have that $v_{b^{*} j}-p_{j}^{*}=$ $v_{b^{*} k}-p_{k}^{*}=v_{b^{*} k}-p_{k} \geq v_{b^{*} q^{\prime}}-p_{q^{\prime}}=v_{b^{*} q^{\prime}}-p_{q^{\prime}}^{*}$, where, in the inequality, we used the competitivity of $p$.

Case 2. $q \in\{j, k\}$. If $q^{\prime} \notin\{j, k\}$, the result is immediate from the competitiveness of $p$. If $q^{\prime} \in\{j, k\}$, then $b \neq b^{*}$, so $b \neq \mu(j)$ and $b \neq \mu(k)$. We have that,

$$
\begin{aligned}
& v_{b q}-p_{q}^{*}=v_{b q}-p q \geq v_{b k}-p_{k}=v_{b j}-p_{j}^{*}=v_{b q^{\prime}}-p_{q^{\prime}}^{*}, \text { if } q^{\prime}=j, \text { and } \\
& v_{b q}-p_{q}^{*}=v_{b q}-p_{q} \geq v_{b k}-p_{k}=v_{b k}-p_{k}^{*}=v_{b q^{\prime}}-p_{q^{\prime}}^{*}, \text { if } q^{\prime}=k,
\end{aligned}
$$

where the inequalities follow from the competitiveness of $p$.

Then, in any case, $v_{b q}-p_{q}^{*} \geq v_{b q^{\prime}}-p_{q^{\prime}}^{*}$. Therefore, $p^{*}$ is competitive. However, $p^{*}<p$, which contradicts the minimality of $p$. Hence, $p_{j}=p_{k}$ and the proof is complete.

Proposition 2. The dynamic mechanism is manipulable by the buyers.

Proof. We have to show that there is a market $(B, Q, v, r, s)$ for which straightforward behavior is not a dominant strategy for some buyer. Then, consider the market where $B=\{1,2\}, Q=\left\{q_{1}, q_{2}, q_{3}, 0\right\}$ and $r(1)=2, r(2)=1$ and $s\left(q_{j}\right)=1$ for all $j=1,2,3$. Buyer 1 values object $q_{1}$ in $7, q_{2}$ in 6 and $q_{3}$ in 4 ; buyer 2 values $q_{1}$ in $8, q_{2}$ in 6 and $q_{3}$ in 3 . If buyers behave straightforwardly, the price obtained in the auction is $(3,1,0)$ and buyer 1 gets $q_{2}$ and $q_{3}$, and buyer 2 gets $q_{1}$. Then, the payoff of buyer 1 will be $(6-1)+(4-0)=9$. Now, let buyer 1 bid as if her vector of values were $(7,6,6)$, instead of $(7,6,4)$. The auction will be conducted as follows:

Step 1. $p(1)=(0,0,0) . \quad B_{1}(1)=q_{1},\left[q_{2}, q_{3}\right]$ and $B_{2}(1)=q_{1}$. The only demand structure is given by $A_{1,1}(1)=q_{1}, A_{1,2}(1)=q_{2}, q_{3}$ and $A_{2,1}(1)=q_{1}$. The minimum overdemanded set is $\left\{q_{1}\right\}$. Then, the auctioneer raises the price of $q_{1}$ by one unit.

Step 2. $p(2)=(1,0,0) . \quad B_{1}(2)=\left[q_{1}, q_{2}, q_{3}\right]$ and $B_{2}(2)=q_{1}$. The demand structure given by $A_{1,1}(2)=q_{2}, A_{1,2}(2)=q_{1}, q_{3}$ and $A_{2,1}(2)=q_{1}$ has no overdemanded set. There is a competitive matching which allocates objects $q_{2}$ and $q_{3}$ to buyer 1 , and $q_{1}$ to buyer 2. (Observe that there are also other demand structures which have an overdemanded set. Consider, for example, $A_{1,1}^{\prime}(2)=q_{1}$, $A_{1,2}^{\prime}(2)=q_{2}, q_{3}$ and $\left.A_{2,1}^{\prime}(2)=q_{1}\right)$.

Buyer 1 will pay nothing for both items. Her true payoff will be $6+4=10>9$. Therefore, straightforward behavior is not a dominant strategy for buyer 1 and hence the auction mechanism is manipulable.

However,

Proposition 3. Suppose the quota of each buyer is the total number of objects. Then, truth telling is a dominant strategy for each buyer. 
Proof. When the quota of the buyers is the total number of objects, a bidder demands some object whenever its price does not exceed her value to the object. Thus, if the price of object $q$ is less than its second highest value, there will be at least two copies of bidders demanding only $q$. Then, object $q$ will be overdemanded at any demand structure. When the price of object $q$ reaches its second highest value, there will be some demand structure such that, at most, one copy of bidder demands only $q$. This bidder has the highest value for object $q$. Any other copy of bidder that demands $q$ will also demand the null object. Thus, at this price, $q$ will not belong to any overdemanded set and its final price will be its second highest value. Therefore, each object will be sold to the bidder who values it most at the price of its second highest value. Thus, each object could be sold independently through $n$ auctions of a single item. Each of these auctions yields the minimum competitive equilibrium for the restricted market that allocates the object to the highest bidder at the price of its second highest value. In addition, each of these auctions is strategically equivalent to the Vickrey's auction, so the compatibility of incentives observed in that auction applies and we have completed the proof.

\section{Concluding Remarks and Related Works}

We proposed a simultaneous ascending bid auction to sell a set of indivisible items to a set of potential buyers. Given a price vector, the demand set of a buyer is determined by her preferences over individual objects at the given price. These preferences are determined by matrix $\left\{v_{b q}\right\}$. Utilities are separable and additive. The markets of art, cars and real estate, for example, fit into this model. The auction is very simple for the buyers:

In each step, they only have to indicate their demands. The rules used by the auctioneer, then, determine the outcome.

In the sequential auctions, similar objects can be sold for quite different prices: Items sold later typically fetch less than items sold earlier (Ashenfelter, 1989, McAfee and Vincent, 1993). Furthermore, a buyer can regret having filled her quota in the early auctions and not having waited for a preferable set of objects offered later.

In the usual sealed bid auctions, buyers must bid blind, unable to know how high they must bid to win a particular item. The buyers take the risk of winning more objects than they need. This risk induces low bidding from budgetconstrained buyers.

The auction described herein is designed so that similar objects are sold for the same price and no buyer receives more objects than she wishes to acquire. In addition, the auction yields the same price vector independent of the auctioneer's choices. The ascending bids allow buyers to see how high their opponents value each object and which set of objects they are looking for. As the final step of the auction approaches, each buyer is able to know which set of objects she is more likely to obtain and, roughly, how much she will have to pay for it. On 
the other hand, at each stage of the mechanism, all objects are open for bidding simultaneously. Thus, a buyer is able to select whatever set of objects she wishes, as well as switch some of the items for others, if their prices become too expensive. Therefore, the ascending bids and the simultaneous bids contribute to efficiency.

The theory developed in this paper shows that, if buyers built their bids thinking that their values are given by matrix $\left\{v_{b q}^{\prime}\right\}$, the final price is the minimum competitive equilibrium price for the competitive market corresponding to $\left\{v_{b q}^{\prime}\right\}$. The final allocation can be chosen so that it is efficient for this market. In this case, it can be easily proved that the dynamic auction is strategically equivalent to a revelation mechanism. This mechanism requires that each buyer select a value to each item. The auctioneer then allocates the objects in accordance with the minimum competitive equilibrium for the competitive market $\left(B, Q, v^{\prime}, r\right)$.

However, this is a very special case. We believe that our dynamic mechanism allows for a much wider range of preferences for the buyers. The results demonstrated here suggest that for small enough bid increases, our mechanism will yield prices approximating the minimum price equilibrium. Nevertheless, our results are given here only for the linear surplus case. For the particular case in which buyers have quota of one, Alkan (1988) presents a dynamic mechanism that finds an equilibrium price in finitely many steps, if utility functions are piecewise linear, and that approximates an equilibrium price for general continuous utilities. Nonetheless, the analysis for more general quotas remains a problem for future investigation and we hope that the model focused here is helpful in building understanding.

As for related works, the general quota model in the context of firms and workers is studied in Sotomayor $(1992,1999)$. A different version is presented in Crawford and Knoer (1981). It is assumed that firms can hire more than one worker and workers can accept more than one job. The main results for this model are that the set of competitive equilibrium payoffs, which coincides with the core, is always non-empty and is a complete lattice.

For the quota one case, the idea to design mechanisms for making allocations with desirable properties of fairness and efficiency has been widely explored in the literature. In the second-price auction, first described by Vickrey (1961), buyers submit sealed bids for a single object, which is then sold to the highest buyer at a price equal to the second highest bid. We can think of the second price auction outcome as an ordinary competitive equilibrium. At the given price, the object is demanded by only one buyer, namely the highest buyer and, as there is only one object, this yields balance of supply and demand. The important property of the second highest bid is that it is the minimum competitive equilibrium price, since for any smaller price at least two buyers would demand the object.

A generalization of the second-price auction of a single item to the buyer-seller market game of Shapley and Shubik is considered by Demange (1982) and Leonard (1983). In their multi-item auction mechanism, each buyer is required to submit 
a sealed bid, listing her valuation for all the items. The auctioneer then allocates the objects in accordance with the minimum competitive equilibrium price.

For the same market, a three-stage auction is presented in Sotomayor (2005): sellers indicate their reservation prices in a first round. Then, each buyer, knowing the choices of the sellers, chooses a value for all items in a second round. The auctioneer allocates the objects in accordance with some market clearing price rule. If no ties exist in the selection of the market allocation, we will have the final allocation. Otherwise, the buyers participate, in the third round, in an auction with a minimum competitive equilibrium price rule, having the price obtained in the second step as the reservation price for the objects. The strategic behavior of buyers and sellers is analyzed under complete information.

Still for the Assignment Game, Sotomayor (2002) proposes a new descending bid method for auctioning multiple objects, which generalizes the Dutch auction and produces the maximum competitive equilibrium price.

Ausubel (1995) presents a mechanism that yields the minimum competitive equilibrium price. In his model, buyers can buy more than one item, goods are homogeneous and consumers have decreasing marginal utilities.

\section{References}

Alkan, A. (1988). Auctioning several objects simultaneously. Bogazici University, mimeo.

Alkan, A., Demange, G., \& Gale, D. (1991). Fair allocation of indivisible goods and criteria of justice. Econometrica, 59(4):1023-1039.

Ashenfelter, O. (1989). How actions work for wine and art. Journal of Economic Perspectives, 3:23-36.

Ausubel, L. (1995). An efficient ascending auction for multiple objects. University of Maryland, Baltimore, mimeo.

Crawford, V. P. \& Knoer, E. M. (1981). Job matching with heterogeneous firms and workers. Econometrica, 49(2):437-450.

Dantzig, G. B. (1963). Linear programming and extensions. Princeton University Press.

Demange, G. (1982). Strategyprofness in the assignement market game. Paris: Ecole Polytechnique, Laboratoire D' Econometrie, Preprint.

Demange, G., Gale, D., \& Sotomayor, M. (1986). Multi-item auctions. Journal of Political Economy, 94(4):863-872.

Gale, D. (1960). The Theory of Linear Economic Models. McGraw-Hill, New York. 
Gul, F. \& Stachetti, E. (2000). The English auction with differentiated commodities. Journal of Economic Theory, 92:66-95.

Hall, P. (1935). On representatives of subsets. Journal of the London Mathematical Society, 10:26-30.

Kelso, A. \& Crawford, V. P. (1982). Job matching, coalition formation and gross substitutes. Econometrica, 50(6):1483-1504.

Leonard, H. B. (1983). Elicitation of honest preferences for the assignment of individuals to positions. Journal of Political Economy, 91:461-479.

McAfee, R. P. \& Vincent, D. (1993). The declining price anomaly. Journal of Economic Theory, 60:191-212.

Roth, A. \& Sotomayor, M. (1990). Two-sided matching. A study in game-theoretic modeling and analysis. Econometric Society Monograph Series, 18. Cambridge University Press.

Shapley, L. \& Shubik, M. (1972). The assignment game I: The core. International Journal of Game Theory, 1:111-130.

Sotomayor, M. (1992). The multiple partners game. In Majumdar, M., editor, Equilibrium and Dynamics: Essays in Honor of David Gale. Macmillan Press Ltd.

Sotomayor, M. (1999). The lattice structure of the set of stable outcomes of the multiple partners assignement game. International Journal of Game Theory, $28: 567-583$.

Sotomayor, M. (2002). A simultaneous descending bid auction for multiple items and unitary demand. Revista Brasileira de Economia, 56(3):497-510.

Sotomayor, M. (2005). Buying and selling strategies in the assignment game. mimeo.

Vickrey, W. (1961). Counterspeculation, auctions and competitive sealed tenders. Journal of Finance, 16:8-37. 


\section{Appendix}

In this section we will demonstrate some of the results stated in section 3 . The following remark will be useful for the proofs.

Remark 3. Let $t$ be some step of the auction at which the set $U(t) \neq \phi$ and $p(t) \leq y$. Let $A(t)$ be any demand structure at step $t$. Write the set of elements of $\mathbf{B}_{\mathbf{b}}(\mathbf{t})$ as $\mathbf{F}(\mathbf{t}) \cup \mathbf{G}(\mathbf{t})$ and the set of elements of $\mathbf{B}_{\mathbf{b}}(\mathbf{y})$ as $\mathbf{F}(\mathbf{y}) \cup \mathbf{G}(\mathbf{y})$, with $G(t) \neq \phi, G(y) \neq \phi, F(t) \cap G(t)=\phi, F(y) \cap G(y)=\phi$, such that $b$ is indifferent between any two objects of $G(t)$ (respectively $G(y)$ ) at price $p(t)$ (respectively $y$ ). In addition, $b$ strictly prefers any object of $F(t)$ (respectively $F(y)$ ), if any, to any object of $G(t)$ (respectively $G(y)$ ) at price $p(t)$ (respectively $y$ ) (Observe that $G(t)$ as well as $G(y)$ may have only one element). It is clear that, for all $j \neq r(b)$, either $\mathbf{A}_{\mathbf{b}, \mathbf{j}}(\mathbf{t}) \subseteq \mathbf{F}(\mathbf{t})$ (respectively $\mathbf{A}_{\mathbf{b}, \mathbf{j}}^{*}(\mathbf{y}) \subseteq \mathbf{F}(\mathbf{y})$ ) or $\mathbf{A}_{\mathbf{b}, \mathbf{j}}(\mathbf{t}) \subseteq \mathbf{G}(\mathbf{t})$ (respectively $\left.\mathbf{A}_{\mathbf{b}, \mathbf{j}}^{*}(\mathbf{y}) \subseteq \mathbf{G}(\mathbf{y})\right)$. Also, $\mathbf{A}_{\mathbf{b}, \mathbf{r}(\mathbf{b})}(\mathbf{t}) \subseteq \mathbf{G}(\mathbf{t}) \quad\left(\right.$ respectively, $\left.\mathbf{A}_{\mathbf{b}, \mathbf{r}(\mathbf{b})}^{*}(\mathbf{y}) \subseteq \mathbf{G}(\mathbf{y})\right)$ and if $\mathbf{A}_{\mathbf{b}, \mathbf{j}}(\mathbf{t}) \subseteq \mathbf{F}(\mathbf{t})$ (respectively $\left.\mathbf{A}_{\mathbf{b}, \mathbf{j}}^{*}(\mathbf{y}) \subseteq \mathbf{F}(\mathbf{y})\right)$ then $\quad\left|\mathbf{A}_{\mathbf{b}, \mathbf{j}}(\mathbf{t})\right|=\mathbf{1}$ (respectively $\left.\left|\mathbf{A}_{\mathbf{b}, \mathbf{j}}^{*}(\mathbf{y})\right|=\mathbf{1}\right)$.

Now, let $C \subseteq Q$. Suppose that $p_{q}(t)=y_{q}$ for all $q \in C, b$ is indifferent between any two elements of $C$ at prices $p(t)$ (and so is at prices $y$ ), $\mathbf{C} \subseteq \mathbf{G}(\mathbf{t}$ ) and $\mathbf{C} \subseteq \mathbf{G}(\mathbf{y})$. Then, $F(t)$ (respectively $F(y)$ ) is the set of all objects that are strictly preferred by $b$ to any object of $C$ at price $p(t)$ (respectively $y$ ). Also, $F(t) \cup G(t)$ (respectively $F(y) \cup G(y)$ ) is the set of all objects that are weakly preferred by $b$ to any object of $C$ at price $p(t)$ (respectively $y$ ). It can be shown that

$$
\mathbf{F}(\mathbf{y}) \cup \mathbf{G}(\mathbf{y}) \subseteq \mathbf{F}(\mathbf{t}) \cup \mathbf{G}(\mathbf{t}) \text { and } \mathbf{F}(\mathbf{y}) \subseteq \mathbf{F}(\mathbf{t})
$$

and

$$
\mathbf{G}(\mathbf{y}) \cap \mathbf{U}(\mathbf{t})=\mathbf{G}(\mathbf{t}) \cap \mathbf{U}(\mathbf{t}) \text { and } \mathbf{F}(\mathbf{y}) \cap \mathbf{U}(\mathbf{t})=\mathbf{F}(\mathbf{t}) \cap \mathbf{U}(\mathbf{t}) .
$$

It is also important to point out that,

$$
\text { if } \begin{aligned}
\mathbf{A}_{\mathbf{b}, \mathbf{i}}(\mathbf{t}) & \subseteq \mathbf{F}(\mathbf{t}) \cap \mathbf{U}(\mathbf{t}), \text { for some }(\mathbf{b}, \mathbf{i}), \text { then } \\
\mathbf{A}_{\mathbf{b}, \mathbf{i}}(\mathbf{t}) & =\mathbf{A}_{\mathbf{b}, \mathbf{j}}^{*}(\mathbf{y}), \text { for some }(\mathbf{b}, \mathbf{j}) .
\end{aligned}
$$

The original proof of Lemma 1 is very long. The details of this proof, as well as of the proof of (A.1), (A.2) and (A.3) of Remark 3, are available from the author upon request.

Sketch of the proof of Lemma 1. Define $A^{\prime}(t)$ as follows. If $(b, i) \notin T^{\prime}$, define $A_{b, i}^{\prime}(t) \equiv A_{b, i}(t)$. Now, suppose that for all $(b, i) \in T^{\prime}$ there is some $(b, j)$, such 
that $A_{b, j}^{*}(y) \subseteq A_{b, i}(t) \cap U(t)$. Then, define $A_{b, i}^{\prime}(t) \equiv A_{b, i}(t)$ for all $(b, i) \in T^{\prime}$ and we are done. Otherwise, there is some $(b, i) \in T^{\prime}$, with $A_{b, i}(t) \equiv C \cup E$, where $C=A_{b, i}(t) \cap U(t) \neq \phi$, such that,

for all $(b, j), A_{b, j}^{*}(y)$ is not contained in $C$. (1)

We have to show that it is possible to define $A^{\prime}(t)$ so that, for all $(b, j) \in T^{\prime}$, and $j \neq r(b)$, there exists some $(b, k)$ such that $A_{b, j}^{\prime}(t)=A_{b, k}^{*}(y) \subseteq U(t)$ and if $(b, r(b)) \in T^{\prime}$, there exists some $(b, k)$ such that $A_{b, k}^{*}(y) \subseteq A_{b, r(b)}^{\prime}(t) \cap U(t)$. The plan of the proof is the following:

1) Define $F(t), G(t), F(y)$ and $G(y)$ as in Remark 3 . Then see that $\mathbf{A}_{\mathbf{b}, \mathbf{i}}(\mathbf{t}) \subseteq \mathbf{G}(\mathbf{t})$, so $\mathbf{C} \subseteq \mathbf{G}(\mathbf{t})$.

2) Observe that $\mathbf{C}$ is contained in the set of elements of list $\mathbf{B}_{\mathbf{b}}(\mathbf{y})$, so $\mathbf{C} \subseteq \mathbf{G}(\mathbf{y})$, otherwise we contradict (1).

3) Then we have satisfied the hypothesis of Remark 3 and so, $\mathbf{F}(\mathbf{y}) \cup \mathbf{G}(\mathbf{y}) \subseteq \mathbf{F}(\mathbf{t}) \cup \mathbf{G}(\mathbf{t})$ and $\mathbf{F}(\mathbf{y}) \subseteq \mathbf{F}(\mathbf{t})$.

4) Observe, using (1), that if $(b, j)$ is such that $A_{b, j}^{*}(y) \cap C \neq \phi$, then $j=r(b)$,

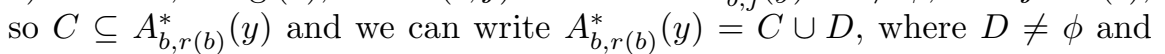
$D \cap C=\phi$. It is clear that $A_{b, r(b)}^{*}(y) \subseteq G(y)$, because $(b, r(b))$ is the last copy of $b$.

5) Set:

$$
\begin{aligned}
\Gamma & \equiv\left\{(b, j) ; A_{b, j}(t) \subseteq G(t) \text { and } A_{b, j}(t) \cap U(t) \neq \phi\right\} \\
\Gamma^{\prime} & \equiv\left\{(b, j) ; A_{b, j}^{*}(y) \subseteq G(y) \text { and } A_{b, j}^{*}(y) \cap U(t) \neq \phi\right\} \\
\mathfrak{F} & \equiv\left\{(b, j) ; A_{b, j}(t) \subseteq G(t) \text { and } A_{b, j}(t) \cap U(t)=\phi\right\} \\
\mathfrak{F}^{\prime} & \equiv\left\{(b, j) ; A_{b, j}^{*}(y) G(y) \text { and } A_{b, j}^{*}(y) \cap U(t)=\phi\right\}
\end{aligned}
$$

We have that $\Gamma \neq \phi$, since $(b, i) \in \Gamma$. Also, $\Gamma^{\prime} \neq \phi$, since $(b, r(b)) \in \Gamma^{\prime}$. The next step is to show that $|\Gamma| \leq\left|\Gamma^{\prime}\right|$ and $(b, r(b)) \in \Gamma$ in order to be able to define a one-to-one map $f$ from $\Gamma-\left\{(b, r(b)\}\right.$ into $\Gamma^{\prime}-\{(b, r(b)\}$.

6) Then define:

$$
\begin{aligned}
\mathbf{A}_{\mathbf{b}, \mathbf{j}}^{\prime}(\mathbf{t}) & \equiv \mathbf{A}_{\mathbf{b}, \mathbf{j}}(\mathbf{t}) \text { if } \mathbf{A}_{\mathbf{b}, \mathbf{j}}(\mathbf{t}) \subseteq \mathbf{F}(\mathbf{t}) \text { or }(\mathbf{b}, \mathbf{j}) \in \mathfrak{F} \\
\mathbf{A}_{\mathbf{b}, \mathbf{j}}^{\prime}(\mathbf{t}) & \equiv \mathbf{A}_{\mathbf{f}(\mathbf{b}, \mathbf{j})}^{*}(\mathbf{y}) \text { if }(\mathbf{b}, \mathbf{j}) \in \Gamma-\{(\mathbf{b}, \mathbf{r}(\mathbf{b}))\} \\
\mathbf{A}_{\mathbf{b}, \mathbf{r}(\mathbf{b})}^{\prime}(\mathbf{t}) & \equiv \mathbf{G}(\mathbf{t})-\cup_{\mathbf{j} \neq \mathbf{r}(\mathbf{b})} \mathbf{A}_{\mathbf{b}, \mathbf{j}}^{\prime}(\mathbf{t})
\end{aligned}
$$


It is a matter of verification that $A_{b}^{\prime}(t)$ is well defined (use $G(t) \cap U(t)=$ $G(y) \cap U(t)$ given by (A.2) of Remark 3).

Now, use Remark 3 again, to see that $A^{\prime}(t)$ is the desired demand structure. Hence the proof is complete.

Proof of Lemma 2. Suppose by way of contradiction that $\mathrm{S}$ is a minimal overdemanded set for $A^{\prime}(t)$ and $S_{1} \equiv S \cap U(t) \neq \phi$. Let $T$ be the set of loyal demanders of $S$. The fact that $S$ is overdemanded means exactly that

$$
|T|>|S|
$$

We will show that $S-S_{1}$ is non-empty and overdemanded for $A^{\prime}(t)$, so $S$ is not a minimal overdemanded set for $A^{\prime}(t)$, which is a contradiction. To see this, define $T_{1}=\left\{(b, i) \in T ; A_{b, i}^{\prime}(t) \cap S_{1} \neq \phi\right\}$. Let $T^{\prime}$ be as defined in Lemma 1. Now, observe that $T_{1} \subseteq T^{\prime}$ and so, by Lemma 1 , for each $(b, i) \in T_{1}$ there is some $(b, j)$, such that $A_{b, j}^{*}(y) \subseteq A_{b, i}^{\prime}(t) \cap U(t)$. On the other hand, the fact that $(b, i) \in T$ implies that $A_{b, i}^{\prime}(t) \subseteq S$, so $A_{b, i}^{\prime}(t) \cap U(t)=A_{b, i}^{\prime}(t) \cap S_{1}$. Then, $A_{b, j}^{*}(y) \subseteq A_{b, i}^{\prime}(t) \cap S_{1}$, so $A_{b, j}^{*}(y) \subseteq S_{1}$. That is, for each $(b, i) \in T_{1}$ there is some $(b, j)$, such that $A_{b, j}^{*}(y) \subseteq S_{1}$. Thus,

$$
\left|T_{1}\right| \leq\left|\left\{(b, j) ; A_{b, j}^{*}(y) \subseteq S_{1}\right\}\right| \leq\left|S_{1}\right|,
$$

where the last inequality is due to the competitiveness of $y$. But then, (1) and (2) imply that $\left|T-T_{1}\right|>\left|S-S_{1}\right| \geq 0$, from which it follows that $T-T_{1} \neq \phi$. However, $T-T_{1}=\left\{(b, i) \in T ; A_{b, i}^{\prime}(t) \subseteq S-S 1\right\}$, so $S-S_{1}$ is non-empty and overdemanded for $A^{\prime}(t)$, as we wanted to show.

Proof of Lemma 3. Suppose that $A^{\prime}(t)$ has overdemanded sets. Let $S$ be some minimal overdemanded set for $A^{\prime}(t)$. Let $T$ be the set of loyal demanders of $S$. Let $T^{\prime}$ be as defined in Proposition 1. By Lemma 2, $S \cap U(t)=\phi$. Then, if $(b, i) \in T$, $A_{b, i}^{\prime}(t) \subseteq S$, so $A_{b, i}^{\prime}(t) \cap U(t)=\phi$, so $(b, i) \notin T^{\prime}$. By Lemma $1, A_{b, i}^{\prime}(t)=A_{b, i}(t)$. Then, for all $(b, i) \in T, A_{b, i}(t) \subseteq S$. Hence, $S$ is also minimal overdemanded for $A(t)$, and the proof is complete.

For the proof of Theorem 2, we need some preliminaries. Consider market $M=(B, Q, v, r)$. We can define a related market in which each buyer $b$ with quota $r(b)$ is "replicated" $r(b)$ times so that each agent has quota of 1 . Denote by $(b, i)$ the $i$ th copy of $b$. Set $B^{*} \equiv\{(b, 1), \ldots,(b, r(b)), b \in B\}$. Let $r^{*}(b, i)=1$ for all $(b, i)$ in $B^{*}$. Let $\mu$ be an optimal matching for $M$. Now, let $\mu^{*}$ be as follows:

if $\mu^{*}(\mathbf{b}, \mathbf{i})=\mathbf{q}$, then $\mu(\mathbf{q})=\mathbf{b}$

if $\mu(\mathbf{q})=\mathbf{b}$, then $\mu^{*}(\mathbf{b}, \mathbf{i})=\mathbf{q}$ for exactly one copy, $(\mathbf{b}, \mathbf{i})$, of $\mathbf{b} .(\mathrm{A} .7)$

Then, if object $q$ is matched to buyer $b$ under $\mu$, this means that object $q$ is matched to some copy of $b$ under $\mu^{*}$ and no other element of $B^{*}$ is matched to $q$. For each pair $(v, \mu)$, there exists one and only one matrix $v^{*}$ defined as follows: 


$$
\begin{aligned}
& \mathbf{v}_{(\mathbf{b}, \mathbf{i})}^{*} \mathbf{q}=\mathbf{0} \text { if } \mu^{*}(\mathbf{q})=\mathbf{b} \text { and } \mu^{*}(\mathbf{q}) \neq(\mathbf{b}, \mathbf{i}) \\
& \mathbf{v}_{(\mathbf{b}, \mathbf{i})}^{*} \mathbf{q}=\mathbf{v}_{\mathbf{b q}} \text { otherwise. }
\end{aligned}
$$

The related market is then $M^{*}=\left(B^{*}, Q, v^{*}, r^{*}\right)$. It is very easy to verify that $\mu^{*}$ is an optimal matching for $M$. The connection between the competitive equilibria for $M$ and $M^{*}$ is given by the following result, which follows from Proposition 2 of Sotomayor (1992).

Proposition $1^{*}$. Let $\mu$ be an optimal matching for $M$. Let $\mu^{*}$ be obtained from $\mu$ by rules (B.1) and (B.2). Let $v^{*}$ be obtained from $v$ and $\mu$ by functional relations (B.3) and (B.4). Then, (i) $p$ is a competitive price for $M$, if and only if $p$ is a competitive price for $M^{*}$ and (ii) $(p, \mu)$ is a competitive equilibrium for $M$, if and only if $\left(p, \mu^{*}\right)$ is a competitive equilibrium for $M^{*}$.

We will make use of the following result that can also be found in Roth and Sotomayor (1990).

Proposition $2^{*}$ Demange et al. (1986). Suppose all buyers have quota of one. Then, if $p$ is the minimum competitive price, there is a matching $\mu^{\prime}$, such that $\left(p, \mu^{\prime}\right)$ is a competitive equilibrium.

Proof of Theorem 2. Let $\mu$ be an optimal matching for $M=(B, Q, v, r)$. Let $\mu^{*}$ be obtained from $\mu$ by rules (B.1) and (B.2). Let $M^{*}=\left(B^{*}, Q, v^{*}, r^{*}\right)$ be the related one-to-one market where $v^{*}$ is obtained from $v$ and $\mu$ by functional relations (B.3) and (B.4). Proposition $1^{*}$ implies that $p$ is the minimum competitive price for $M^{*}$. By Proposition $2^{*}$ there is a matching $\mu^{\prime}$, such that $\left(p, \mu^{\prime}\right)$ is an equilibrium for $M^{*}$. Since $\mu^{*}$ is optimal for $M^{*}$, it is compatible with any competitive equilibrium price. Thus, we obtain that $\left(p, \mu^{*}\right)$ is a competitive equilibrium for $M^{*}$. By Proposition $1^{*}$ again, $(p, \mu)$ is an equilibrium for $M$ and the proof is complete. 